\title{
Las representaciones sociales a bordo del automóvil en Medellín, Colombia*
}

\author{
Felipe Vallejo Uribe ${ }^{* *}$ \\ Zulima Azeneth López Torres ${ }^{\star \star *}$ \\ Recibido: 2018-09-02. Enviado a pares: 2018-09-15. \\ Aprobado por pares: 2018-11-03. Aceptado: 2018-11-28 \\ https://doi.org/10.22395/angr.v17n34al 1
}

\begin{abstract}
Resumen
Más allá de su valor monetario, los automóviles privados tienen un valor social que los mantiene en uso a pesar de las restricciones económicas y ambientales que pesan sobre ellos. Esta investigación analiza las representaciones sociales que se construyeron en Medellín (Colombia) alrededor del vehículo particular y por qué este bien de lujo sigue creciendo en su uso a pesar de que la ciudad presenta alternativas públicas de movilidad. Se exploró la bibliografía existente sobre las representaciones sociales, especialmente de los bienes de lujo, además se desarrollaron entrevistas semiestructuradas a un grupo de personas entre 30 y 40 años que poseen vehículos para uso particular que al momento de su compra costaron al menos 50 millones de pesos colombianos. El análisis del discurso de los entrevistados permitió conocer el valor social que las personas dan al vehículo privado. Esta valoración que los propietarios dan a ese bien va más allá de los conceptos de libertad e independencia que reconoce la literatura relacionada, este ejercicio mostró que el primer objetivo de los poseedores es desligarse de los sistemas de transporte público o colectivo. Los poseedores no solo comprenden el valor simbólico del automóvil, sino que anticipan las reacciones que causa en los demás y lo utilizan para construir un discurso y mostrarse como desean ante otros miembros de su comunidad. Este análisis desde lo sociológico expuso que el consumo de automóviles entre este grupo de estudio se da en una escala ascendente que se relaciona directamente con el estatus aspirado por el propietario del vehículo.
\end{abstract}

Palabras clave: estatus social; transporte; automóvil; identidad; diferenciación social; lenguaje simbólico; propiedad privada; motivación.

Este artículo se origina como trabajo de grado para optar por el título de magíster en Comportamiento del Consumidor de la Universidad Pontificia Bolivariana, sede Medellín. La investigación se denominó Las representaciones sociales a bordo del automóvil privado en la ciudad de Medellín.

* Estudiante de la Maestría en Comportamiento del Consumidor de la Universidad Pontificia Bolivariana, especialista en Gestión de Proyectos del SENA, Medellín y comunicador social-periodista de la Universidad de Antioquia. felipe. vallejo@revistavec.com

*** Psicóloga, magíster en Antropología, coordinadora de la línea Entorno y Consumo del grupo de investigación Epilion de la Facultad de Publicidad de la Universidad Pontificia Bolivariana.zulima.lopez@upb.edu.co. Orcid: https://orcid. org/0000-0001-8558-3556 


\title{
Social representations on board the car in Medellín, Colombia
}

\begin{abstract}
Beyond their monetary value, private cars have a social value that keeps them in use despite the economic and environmental restrictions upon them. This research analyzes the social representations that were built in Medellin (Colombia) around the private vehicle, and why is the use of this luxury good increasing even though the city boasts public transport alternatives. The existing literature on social representations, especially of luxury goods, was reviewed, and semi-structured interviews were conducted with a group of people between 30 and 40 years old who own vehicles for private use that cost at least 50 million of Colombian pesos at the time of their purchase. The discourse analysis of the interviewees allowed to know the social value that people give to private cars. The owners' assessment of that good goes beyond the concepts of freedom and independence as recognized by the related literature. This research showed that the owners' first goal is to distance themselves from public transport systems. Car owners understand the car's symbolic value and foresee the reactions that the good cause on other people, and they use it to build a speech and show themselves as they want to other members of their community. From a sociological point of view, this analysis revealed that car consumption among the aforementioned group of study presents itself an ascending scale that is directly related to the status aspired by the car's owner.
\end{abstract}

Keywords: social status; transport; car; identity; social differentiation; symbolic language; private property; motivation.

\section{As representações sociais a bordo do automóvel em Medellín, Colômbia}

\begin{abstract}
Resumo
Mais além de seu valor monetário, os automóveis privados têm um valor social que os mantêm em uso apesar das restrições econômicas e ambientais que pesam sobre eles. Esta pesquisa analisa as representações sociais que foram construídas em Medellín (Colômbia) ao redor do veículo particular e porque esse bem de luxo continua tendo um uso crescente apesar de a cidade apresentar alternativas públicas de mobilidade. Explorou-se a bibliografia existente sobre as representações sociais, especialmente dos bens de luxo, e desenvolveramse entrevistas semiestruturadas com um grupo de pessoas entre 30 e 40 anos que possuem veículos para uso particular que, no momento da compra, custaram pelo menos 50 milhões de pesos colombianos. A análise do discurso dos entrevistados permitiu conhecer o valor social que as pessoas dão ao veículo privado. Essa valorização que os proprietários dão a esse tipo de bem vai mais além dos conceitos de liberdade e independência reconhecidos pela literatura relacionada. Este exercício mostrou que o primeiro objetivo dos proprietários é se desligar dos sistemas de transporte público ou coletivo. Os donos não somente compreendem o valor simbólico do automóvel, mas também antecipam as reações que causam nos demais e o utilizam para construir um discurso e mostrar-se como desejam diante dos outros membros de sua comunidade. Esta análise a partir do sociológico expôs que o consumo de automóveis entre esse grupo de estudo se dá em uma escala ascendente que se relaciona diretamente com o status aspirado pelo proprietário do veículo.
\end{abstract}

Palavras-chave: status social; transporte; automóvel; identidade; diferenciação social; linguagem simbólico; propriedade privada; motivação. 


\section{Introducción}

Las principales capitales colombianas han atravesado por fuertes crisis ambientales entre 2015 y 2017. Desde 2016, el país ha sentido una desaceleración económica marcada por el aumento de impuestos y de la inflación, las encuestas Origen-Destino del Área Metropolitana del Valle de Aburrá (AMVA) demuestran que los tiempos de desplazamiento han aumentado en la principales capitales, pero aun así la tasa de motorización en Colombia (cantidad de autos por cada 100 mil habitantes) se duplicó en los últimos 10 años. En 2016 había 5,2 automóviles por cada 1.000 habitantes en Colombia, no obstante, esta es una de las tasas de motorización más bajas de América Latina pues Chile y Argentina están en 17,6 y 16,1 respectivamente (BBVA Research, 2017).

A pesar de decrecimiento en el consumo, las cifras de ventas de autos nuevos no han alcanzado los niveles de peligro vistas en sectores como la confección y los servicios. Según la Asociación Colombiana de Vehículos Automotores (Andemos), en Colombia se matricularon 283.380 automóviles en 2015, 253.698 mil en 2016 y en 2017 fueron 238.238 unidades (Andemos, 2017). Esta caída de 10,5 \% y 3,1 \% en los números es muestra de que la economía colombiana redujo su fuerza, pero no niega que todavía los autos son una industria fuerte en Colombia y un bien importante para los ciudadanos. Esto sin contar la venta de autos usados que aumentó en más del 7 \%, hasta 782,4 mil unidades en 2016 (BBVA Research, 2017).

En Antioquia se vendió el 21,6 \% de los autos nuevos que se comercializaron en Colombia durante 2016, el centro de esta región tuvo una crisis ambiental en 2015, otra en 2016 y estuvo a punto de tener dos crisis en 2017. Aun así, las ventas de autos en esa región rondan las 60.000 unidades en cada uno de esos años (Andemos, 2017). Este problema ambiental se visualizó por lo menos desde 2003 y se convirtió en política pública en la ciudad (Alcaldía de Medellín, Secretaría del Medio Ambiente. 2003).

Aunque los propietarios están al tanto de los inconvenientes, una parte notoria de ellos sigue destinando sus ingresos a comprar carro nuevo. Este bien duradero, ha tenido relevancia dentro de la vida de las personas, a pesar de las acciones del gobierno para desincentivar el uso del automóvil particular, promover el transporte público y los medios de transporte no motorizado o limpio en las principales ciudades del país. (Morales, 2014).

Detrás de la utilidad práctica del automóvil, hay un poder simbólico que hace que muchos colombianos sigan deseando un vehículo particular. Poseer un auto es cada vez más complicado, sobre todo por los costos, pero el consumo de autos, como el de cualquier otro bien, es un acto público que nunca será atomizado o pasivo, o sea que es un acto "eminentemente social, correlativo y activo", además es "un impulso socialmente regulado y generado" (Appadurai, 1991, p. 48).

El conjunto de símbolos establecidos alrededor de los autos ayuda a darle sentido a las interacciones que las personas tienen sobre ese objeto. Para Mead (1934), el significado de una conducta se construye a través de la interacción social ya que el individuo perte- 
nece a una estructura social a la cual responde. En su interpretación del interaccionismo simbólico, Mead es todavía más tajante al aseverar que los individuos dependen de una comunidad para ser ellos, sobre todo cuando considera que no pueden tener derechos a menos que se tenga actitudes comunes.

Cada uno de esos 60.000 objetos funcionan sociológicamente como "un medio que, más allá de sus funciones precisas permite evocar creencias, historias singulares e imágenes colectivas" (Martínez, 2006, p. 21). Para entender esta máquina es necesario conocer los motivos por los cuales los ciudadanos la adquieren e invierten grandes sumas en su adquisición y mantenimiento, para esto es necesario conocer las representaciones culturales en las que están envueltas, "no es posible comprender una tecnología sólo por sus propiedades físicas. En el uso, las herramientas entran en relaciones específicas con quienes las usan (...) el instrumento humano expresa una habilidad inconsciente -simbolización-" (Sahlins, 1977, p. 95).

Las normas para seleccionar un vehículo ya están dictadas por el grupo, lo que hace que poseer un automóvil sea un acto social en la mayoría de los casos y se ubique entonces por fuera de la conciencia individual. Emile Durkheim (1986) denominó estos conjuntos de sentidos preestablecidos como representaciones colectivas. Moscovici y Duveen (2000) conceptualizaron un siglo después esta tendencia agrupar significados en las representaciones sociales. Para este psicólogo rumano las representaciones tienen dos características, la primera es que convencionalizan objetos, personas o eventos que encontramos. Ellas dan a estos objetos una forma definitiva, los ubican en una categoría específica y gradualmente los establecen como modelos. La segunda característica es que "son prescriptivas, o sea que se imponen sobre nosotros con una fuerza insuperable. Así, ninguna mente es libre de los efectos del condicionamiento impuesto por las representaciones sociales, el lenguaje y la cultura. (Moscovici y Duveen, 2000, p.64). Las personas perciben lo que las convenciones y la cultura les permiten ver, sin percatarse de la existencia de las representaciones, inclusive Moscovici y Duveen (2000, p. 64) las comparan con ondas de radio que envuelven a las personas llevando información sin que ellas se enteren de su presencial, solo de su interpretación final. Los individuos se relacionan con los objetos por medio del acto de representación social, que funciona como esa interpretación final de la señal de radio.

Las representaciones sociales alrededor del automóvil pueden explicar la necesidad y la admiración que los habitantes de ciertas capitales colombianas tienen frente a ese bien, así, "lo material no tiene de por sí sentido alguno, necesita de la mente y del sentimiento humano para adquirir sentido" (Martínez, 2006, p. 40). El automóvil es también un espejo en el cual sus propietarios, usuario o deseadores se miran, este, como otros objetos, es un "vínculo con cada uno de ellos con ellos mismos y, a su vez, con los demás, vínculo construido a través de referencias propias y cambiantes" (Martínez, 2006, p. 96). Estos significados públicos se sustentan y se repiten por los miembros de una comunidad a través de los rituales, ellos son la principal forma en que se desarrolla a comunicación entre ellos (Douglas, Isherwood y Mercado, 1990). 
Las representaciones sociales deben describirse y analizarse en ambientes y culturas dentro de las que se producen, además los rituales y la tecnología, deben evaluarse en términos de la cultura en la que se conciben y se utilizan (Childe, 1984). En este caso se planteó analizar las representaciones sociales del automóvil en Medellín. Esta es la segunda ciudad de Colombia donde más autos se venden después de Bogotá (Andemos, 2017). Como parte del AMVA, Medellín viene soportando unas crisis ambientales repetitivas desde 2015, como lo titularon los medios de comunicación "La región metropolitana del Valle de Aburrá ha sido incluida en estudios de la Organización Mundial de la Salud (OMS) como una de las diez zonas más contaminadas de Latinoamérica" (El Colombiano, 2016).

Según la Secretaría de Tránsito y Transporte de Medellín, en la ciudad había 1,27 millones vehículos matriculados en 2015. En Medellín, había al terminar 2015 un total de 639.231 motos y 633.993 carros, esto significa que entre 2012 y 2015 su parque automotor circulante aumentó en 15,17\% (Programa Medellín Cómo Vamos, 2016). Además, el Inventario de Emisiones Atmosféricas del Valle de Aburrá determinó que 80\% de las emisiones contaminantes de la ciudad provienen de fuentes móviles (Área Metropolitana del Valle de Aburrá. 2015)

Este crecimiento acelerado, aun en contra de las condiciones de vida de los ciudadanos, radica en gran parte en las representaciones sociales que han anclado a los habitantes de Medellín a los vehículos particulares. Los autos siguen siendo populares en Medellín, cada día ingresan 385 vehículos nuevos al Área Metropolitana del Valle de Aburrá, aunque pesen sobre ellos restricciones, se hayan aumentado los impuestos y la malla vial de la ciudad no se incremente ¿Cómo se configuran entonces las representaciones sociales que mantiene a los habitantes de Medellín unidos a los vehículos particulares propulsados por motores de combustión interna a pesar de las restricciones?

Al revisar las publicaciones anteriores realizadas en Colombia se nota que algunos autores han indagado sobre los automóviles y los motivos que algunos grupos de colombianos tienen para adquirirlos usando enfoques desde la neurociencia y el mercadeo, pero no desde la sociología o las representaciones sociales. Según Martín Juez (2002), esta profundización en necesaria ya que para las personas "un objeto siempre será la expresión legítima de un modo de vivir y ver el mundo". Se puede resaltar a Pedro Luis Duque (2014) quien ve en el "neuromarketing una herramienta validadora en la toma de decisiones en mercadeo visual-auditivo", y desarrolla una investigación cuantitativa sobre las siete marcas de autos más vendidas en Colombia. Igualmente es necesario resaltar la investigación sobre el Valor percibido de vehículos de lujo: dimensionalidad e implicaciones en mercadeo (Durán, Chacón, Ortegón y Atehortúa, 2014), que entrega una mirada desde el mercadeo. Estos trabajos fueron puntos de referencia que ayudaron a crear un paradigma que permitiera comprender el automóvil particular desde sus representaciones sociales. 


\section{Metodología}

En esta búsqueda del sentido del automóvil privado en Medellín, se realizó una investigación cualitativa que partió de la conversación con un grupo de 12 entrevistados entre los 40 y 50 años de edad y que habían comprado un carro de, al menos, 50 millones de pesos colombianos. Este fue un proceso de interpretación hermenéutica de las interacciones de carácter simbólico a través de entrevistas. También se planteó un acercamiento desde el interaccionismo simbólico para comprender la conducta humana alrededor de ese bien llamado automóvil privado, ya que los seres humanos siempre actúan en relación a los objetos del mundo físico partiendo de los significados que esos objetos han desarrollado gracias a la interacción social (Blumer, 1954).

Se creó una guía para desarrollar entrevistas semiestructuradas a una muestra intencional de 12 informantes voluntarios. Esta guía se basó en la bibliografía analizada anteriormente, buscando comprobar si las representaciones sociales encontradas en ella aplicaban para un territorio como Medellín, pero además buscando encontrar posibles nuevas representaciones desarrolladas en esta ciudad. La bibliografía permitió crear categorías. El tamaño de esta muestra lo definió el principio de saturación teórica, y se llegó a conclusiones cuando las respuestas coinciden o se respaldan entre sí.

Todos los entrevistados cumplían con ser personas de 30 a 40 años. Todos los participantes debían haber adquirido en los últimos cinco años un vehículo particular de más de 50 millones de pesos que usaran con fines de transporte particular, lo que determinó que la persona había atravesado por una decisión de compra de un vehículo recientemente. Se procuró que esta muestra tuviera la misma porción de mujeres y de hombres con el fin de localizar posibles diferencias en cada género. Las entrevistas se hicieron en privado, evitando elementos que pudieran sesgar las respuestas de las personas. Los entrevistados no tenían ninguna relación entre sí. Las entrevistas semiestructuradas buscaron que las personas convocadas hablaran sobre cuatro categorías: la primera se denominó individuo, dirigida a la caracterización del entrevistado para construir perfiles de análisis. La segunda se llamó iniciación, dedicada a la reconstrucción de la decisión de compra de su primer vehículo propio con su propio dinero, no vehículo familiar u obsequiado. La tercera fue actualidad, una reconstrucción de la decisión de compra de su último vehículo. La cuarta y última se definió como futuro, y se enfocó al vehículo anhelado y los motivos de deseo hacia este.

El paso final fue sistematizar las respuestas de los entrevistados en categorías emergentes. La imagen que tienen de su automóvil particular, la percepción de su importancia dentro de su vida diaria, la opinión propia y ajena sobre este bien frente a sus diferentes gamas, y la actitud que mostró al adquirir su auto, al usarlo y frente a la situación hipotética de perderlo.

Este estudio partió de los elementos que componen las representaciones sociales creadas alrededor de los automóviles particulares en la cultura de Medellín, este levantamiento teórico aportó a comprender cómo el automóvil trasciende lo utilitario para 
convertirse en un artefacto cultural al servicio de la identidad personal del medellinense, como ya ocurrió en otras ciudades (Urry, 2004).

\section{Resultados}

Las entrevistas, en primer lugar, indagaron a los participantes por su primer auto propio, el objetivo fue encontrar cómo empezaron a hacer parte de los rituales sociales alrededor del automóvil. En los años en que las personas del grupo iniciaban su vida adulta, el transporte público era aún menos eficiente que el actual. El principal motivo para conseguir un vehículo propio fue la insatisfacción con el transporte público en Medellín, esta situación era vista por los entrevistados como una limitante para su libertad, un constrictor de su interacción con otras personas y un obstáculo para el desarrollo de sus aspiraciones. Uno de los entrevistados resumió así el cambio que hubo en su vida cuando pudo tener su primer vehículo propio: "el cambio total fue el poder decir en qué momento podría desplazarme sin tener el obstáculo del tiempo, del servicio público" (Entrevistado 04). Esto se debe a que los sistemas de transporte de las ciudades pueden hacer a las personas prisioneras, aun si se desplazan por grandes distancias (Kaufmann, 2002). Sheller (2004) usó el término "auto-libertad" para definir el inicio de ese amor por los autos y la conducción, que es opuesto al sentimiento de rabia por el tráfico, el aburrimiento por usar las mismas rutas y el disgusto hacia las políticas de transporte del gobierno. Es notorio que la primera sensación de libertad se tiene frente a los sistemas de transporte público rígidos, más que frente a las estructuras de poder como las que se dan en la familia ( Giucci, 2007).

En esta primera parte de cada entrevista también se les pidió a los participantes que describieran qué le faltaba a su primer vehículo propio. La búsqueda de su propia movilidad llevó a todos los entrevistados a aceptar renuncias en capacidades técnicas y estéticas de sus vehículos, a esto se suma el desconocimiento técnico y la menor capacidad adquisitiva pero les permitía forjar mejor una identidad, crear un microambiente que los protegiera, los identificara y los guiara hacia donde quieren estar en uno años. Paul Graves-Brown (2000) se acerca a pensar que los autos privados son hábitats y son también pieles, el primero lo aísla del mundo, mientras que la segunda le ayuda al conductor a protegerse y a proyectar una imagen en el espacio que ocupa.

Avanzando en las entrevistas se llegaba al vehículo que las personas conducían actualmente. Con el paso de los años y la práctica de adquirir autos, menos gente es necesaria en los procesos de decisión. En esta etapa, los autos son conseguidos más intencionalmente hacia unas necesidades personales, necesidades que configuran el estilo de vida del propietario. El primer automóvil de los entrevistados funcionó como un entrenamiento para entender los arquetipos usados por las marcas en sus diseños. Los usuarios saben cada vez mejor cómo aprovechar para su propia identidad la reputación y el lenguaje de los fabricantes. Los productores de autos manipulan el deseo hacia la marca a través de la resonancia emocional de sus campañas publicitarias (Sheller, 2004). Dentro de la imaginación de los anuncios publicitarios hay aspectos que los conductores 
recogen y reutilizan en la comunicación de su identidad. Grant McCracken presenta como responsable de este proceso hoy a dos instituciones de transferencia: la publicidad y el diseño de producto (que también puede llamarse moda). El primero es un ejercicio que junta las características físicas de un objeto y su significado cultural, para así construir una identidad que permita al consumidor crear una imagen de ese bien o servicio, el segundo es la manipulación de los valores simbólicos de un bien a través de algún influenciador (McCracken, 1986).

En esta segunda parte de las entrevistas los participantes mostraron una rápida y clara interpretación de las formas y volúmenes de los autos. Se tendió a asignar a cierto tipo de personas un tipo de auto: los jóvenes usan hatchback, las personas serias y maduras usan sedanes, y quienes son aventureros usan camionetas. Esta tendencia de definirse a sí mismo y a las demás personas por el tipo de carro que tienen fue latente en todas las entrevistas. John Urry (2003) resalta esta relación entre las formas de los autos y las personas, él argumenta que los vehículos son extensiones del conductor. Así, cada contorno o forma del auto es relacionada a su vez con una característica de su conductor. Este conjunto de significados construidos alrededor del automóvil fue usado por los entrevistados para manifestar los cambios de edad. Cambiar de vehículo hacia modelos con otras características aparece como un ritual que marca el paso de la juventud a la adultez, y luego a la madurez.

El primer elemento que resaltaban a la hora de describir su vehículo era la marca. Antes de referirse al nombre del modelo o a sus atributos físicos o técnicos se hablaba de la marca del automóvil seleccionado. La confianza entregada a estas marcas funcionaba como un marco teórico que sustentó todas las decisiones posteriores. Pocos entrevistados sabían claramente cuánta potencia tenía su auto, ninguno supo cuánta seguridad pasiva o activa le entregaba o qué tan alto era su nivel de emisión de gases, pero todos confiaban en que eran objetos técnicamente bien creados que eran suficientes en cada uno de estos aspectos. Como en muchos bienes, las marcas se usan como marcos de referencia para definir los productos que nacen de ellas.

El segundo elemento al que se referían al describir su automóvil propio fue su forma y volumen. Fácilmente y sin que se les preguntara directamente, los entrevistados adjudicaban edades, rasgos de la personalidades o actitudes a ciertos tipos de carrocería, los vehículos más pequeños eran adjudicados a personas más jóvenes, solteros y con pocas responsabilidades. Los vehículos medianos se entregaron a personas maduras, pero con actitudes todavía juveniles. Los autos grandes y poderosos se referenciaron con personas mayores, ocupadas y con familias amplias. En el caso de esta investigación, el entrevistado 08 fue ejemplo de esto, cuando admitió que seleccionó su auto porque le permitía comunicar a las personas a su alrededor "Que es un persona joven, o de espíritu juvenil, aunque no joven en edad, es una persona que no tiene hijos". Este mismo entrevistado admitió que le "gusta más porque es más juvenil, los sedanes nos parecen carros como de mamá, sin actitud". 
La totalidad de los entrevistados admitió que el vehículo que una persona conduce se relaciona con su éxito profesional y la generación de dinero. Entre las entrevistas fue notorio que el automóvil privado se asocia hoy con un grupo de referencias simbólicas, como libertad, velocidad, familia, seguridad, éxito profesional, masculinidad y fuerza. Después de la vivienda, el auto es el bien que más estatus social entrega a su tenedor (Sheller, 2004).

Los entrevistados dejaron claro que los autos deben ser tan buenos como ellos quieren ser, "a medida que tengas más poder adquisitivo vas a querer mejorar en todo, en potencia, velocidad. Cada vez vas a ser más exigente" (Entrevistado 05). El automóvil privado no solo se constituye como un objeto que debe acompañarlos en su edad y posición económica actual, sino que se relaciona con la edad y el poder adquisitivo que aspira tener. El vehículo particular, además de ser una recompensa, aparece como un medio que guía el camino hacia la posición social deseada de su propietario.

Otro momento del cuestionario buscaba saber si los conductores conocían el nivel de emisión de gases contaminantes de su auto o si por lo menos lo tuvieron en cuenta a la hora de realizar la compra. Nadie conocía este dato, pero gran parte asumían que las marcas, porque ser modernas e innovadoras, ya tenía eso resuelto y producían motores de bajas emisiones, hasta llegaron a llamarlos "ecológicos". En una entrevista paralela que se hizo a uno de los pocos diseñadores de autos eléctricos en Colombia, el ingeniero Jorge Barrera argumentó que antes era permitido fumar en todos lados, incluyendo aviones y hospitales, pero la cultura cambió, pronto llegará el día en que dejará de ser socialmente aceptado fumar con los escapes de los automóviles por la ciudad.

La tercera parte del cuestionario se refería a un momento hipotético, en este punto se le pidió al entrevistado hablar de su automóvil soñado. Lo más notorio fue que siempre se quiere más, ya sea en potencia, valor, tamaño, lujos o accesorio. Aparece entonces una continuación de los rituales que tienden a ser más complejos. Cada que se considera la posibilidad de comprar un vehículo nuevo se contempla la opción de adquirir un compromiso económico más importante a través de un vehículo más complejo, especializado y de marcas más exclusivas.

\section{Discusión y conclusiones}

El proceso investigativo con las personas permitió conocer qué uso social les daban a sus vehículos particulares e, incluso, a los de las demás personas. Al desarrollarse quedó claro que entre ellos existe un lenguaje común sustentado por las formas y las marcas de sus autos, ya que los vehículos lograron crear entre ellos un conjunto de códigos que pueden ser leídos tácitamente. Los colores, las dimensiones, la procedencia, los volúmenes y las virtudes de estas máquinas son, en este caso, las palabras que componen este lenguaje de los entrevistados. Al analizar las narraciones entregadas por los entrevistados, de este conocimiento tácito creado alrededor de los carros se puede condensar la información en tres categorías: motivación, identidad personal y aceptación. 
La motivación que las personas tuvieron para adquirir un automóvil particular empezó desde la necesidad de alejarse del transporte público, su objetivo era acercarse a modos de vida más libres y más ceñidos a sus necesidades sociales. Detrás de las motivaciones económicas y de tiempo estuvo la motivación social. Al poseer un vehículo lo primero que comunicaban era que podían seleccionar sus actividades con mayor facilidad y, sobre todo, responsabilidad. En todos los entrevistados el automóvil aparece como el elemento que diferencia una época en su vida, que los "saca" de una juventud y los incrusta en la adultez, ya que les permite un mundo más amplio, no solo en distancias sino en la cantidad de experiencias a las que tiene acceso.

Ese primer vehículo les permitió a los entrevistados empezar a hacer parte de los rituales, de los que ya habían participado como espectadores, no como protagonistas. En este momento llegó la oportunidad para adoptar una de estas máquinas que, aunque no comprendían técnicamente, si comprendían socialmente.

El vehículo inicial fue para la mayoría algo circunstancial, el poder adquisitivo inicial de cuando eran más jóvenes los llevó a aceptar renuncias en cuanto a los atributos mecánicos y físicos. Aun así, todos aceptaron que este vehículo, a pesar de sus deficiencias, los investía con capacidades superiores a las que tenían antes de poseerlo.

Al poner a las personas en el caso hipotético de la desaparición de la posibilidad de usar un vehículo particular, cada uno respondió que esto sería traumático para su vida. El automóvil privado se convierte en el posibilitador de su trabajo y de sus actividades diarias, pero quedó más claro que lo que en verdad posibilita es su estilo de vida. Este apego al vehículo particular pone a los medellinenses en una escalera que solo puede ser usada hacia arriba. Hay un miedo a romper la cadena del consumo de automóviles, una de sus preocupaciones es que el vehículo que adquieran no fuera difícil de vender a otra persona cuando consideren que su ciclo ya acabó. Es necesario adquirir marcas, colores y formas que sean comunes para facilitar que ese vehículo pueda ser vendido o entregado a otra persona y así poder continuar el camino en la escalera. Esta necesidad de renovación incluye a los vehículos dentro del concepto de la moda.

La identidad personal es la segunda categoría en la que se agruparon los resultados. En este punto, las personas entrevistadas mostraron que no solo comprendían el valor simbólico del automóvil, sino que podían anticipar las reacciones que este podría causar en los otros, y así usarlo dentro de su discurso para comunicarse con otros y mostrarse como desea ante los miembros de su comunidad. El automóvil particular, como muchos otros bienes, reduce y condensa conceptos que le permiten ir más allá de la simple capacidad de ubicar a alguien en una clase social. Desear o adquirir cierto automóvil es para este grupo de personas un gesto social, una mezcla entre la experiencia y la conciencia hacia a ese objeto.

A través de la marca, la forma y el volumen del auto empieza una evaluación y una interpretación de la realidad elaborada por el poseedor del vehículo. Por medio de estos 
tres elementos se crea una aproximación del nivel de ingresos, la madurez y la actitud de quien conduce el auto. Aunque casi la totalidad de los entrevistados iniciaron con autos de fabricación nacional y de dos puertas, ninguno quiso regresar a ellos en sus siguientes elecciones, sobre estos recae la idea de que son para personas de menores ingresos y que pertenecen a personas menos maduras laboral y económicamente. Los entrevistados desistieron de este tipo de autos porque ya no simbolizan el nivel social o salarial en que buscan estar ahora y, sobre todo, en el que quiere ser reconocidos. Por esto, se enfocaron en modelos más voluminosos y de menor participación dentro del mercado nacional, o sea, más exclusivos.

Más allá de tener un automóvil particular está la pregunta ¿qué tipo de auto? El tipo de carrocería y color que selecciona el propietario altera la actitud social de este. Cada tipo de vehículo amplía la simbología y enriquece su interpretación. En el ejercicio se encontró que varias personas usan un tipo de auto que no consideran el más apropiado para su vida diaria pero que les ayuda a mostrar un estilo de vida más juvenil, aventurero o elegante. Así, los autos particulares actúan como controladores de la realidad.

Para cada uno de los participantes es claro que se puede relacionar el vehículo que una persona conduce con su éxito profesional, pero una parte del grupo hizo una salvedad en la que reconocen también que las actitudes sostenibles o de gran conciencia social son también muestra de estatus. Dejar de usar el automóvil particular voluntariamente, seleccionar la bicicleta o el ir a pie aparecieron como una bifurcación al hallazgo. Aparece entonces la posibilidad de renunciar a los autos como una actitud por encima de estatus que genera el tener un automóvil y es decidir no usarlo o adquirir uno que no contamine. Resulta como un segundo nivel el reconocimiento social que entrega la movilidad con responsabilidad social o ambiental. Aun así, solo una de las personas admitió que su carro soñado era un vehículo eléctrico.

Este ejercicio entregó mejores resultados cuando se indagó entre las personas del grupo por el vehículo que pudieron comprar al alcanzar un poder adquisitivo mayor. Desde este punto, la selección de la marca, el tipo de carrocería, el color y tamaño fue menos circunstancial y más objetiva, lo que reveló mejor las actitudes sociales de estas personas. En esta etapa ya la imagen de cada vehículo fue absorbida por los propietarios y utilizada como elemento de su identidad personal, sobre todo para denotar cómo es su vida en lo profesional y familiar.

La aceptación social que tienen los vehículos particulares fue una tercera categoría de análisis. En este ejercicio se mostró que entre la población analizada no es importante tener un vehículo que no contamine. Evidentemente, el vehículo particular es considerado como un riesgo para las personas solo en lo relativo a la accidentalidad, pero no se considera su uso como algo contraproducente para la salud. Aunque se reconoce como un objeto contaminante, sobre este no hay ninguna desaprobación frente a su uso. No se condena esa capacidad de emitir gases contaminantes porque todavía no es socialmente desaprobado. 
Sobre los autos el ritual más importante no es comprar un vehículo particular, este es solo la expresión monetaria de una cadena de rituales que posiblemente empiezan mucho antes, desde la infancia, cuando los niños juegan con autos de juguete, pasan por la adolescencia cuando se cuelga el afiche del vehículo soñado en la habitación, acompañan el paso a la adultez con la libertad que entrega aprender a conducir. En cada uno de estos momentos el potencial simbólico de ese objeto se está transfiriendo a través de los individuos, eternizando las representaciones sociales del automóvil.

Dentro del grupo de elementos en que podría ramificarse de esta investigación podría destacarse una profundización en la necesidad de los ciudadanos de Medellín en desligarse de los sistemas de transporte público, conocer las representaciones sociales que se crearon alrededor de ellos podría dar explicaciones al aumento del parque automotor en esta misma ciudad, sobre todo de motos. Otro aspecto viable en el que podría continuar este tema sería cómo los propietarios ven nuevas tecnologías de movilidad, como las eléctricas, sobre las cuales ya se ha estudiado en ciudades como Bogotá (Hennessey. 2014) y Mexico D.F. (Carrete y Arroyo, 2013), y si estas aportan a modificar las representaciones sociales que ya existen alrededor del automóvil particular.

\section{Referencias}

Alcaldía de Medellín, Secretaría del Medio Ambiente. (2003). Formulación del Plan Ambiental municipal -PAM- y diseño del modelo del Sistema de Gestión Ambiental Municipal -SIGAM- Etapas 1y 2. Consorcio Corporación GAIA - Francisco Correa B. Medellín.

Appadurai, A. (1991). La vida social de las cosas. México: Editorial Grijalbo, S.A. de C.V.

Área Metropolitana del Valle de Aburrá. (2015) Inventario de Emisiones Atmosféricas del Valle de Aburrá, año base 2013. Universidad Pontificia Bolivariana, Grupo de Investigaciones Ambientales. Recuperado de www.Metropol.cov.co

Asociación Nacional de Movilidad Sostenible (Andemos). (2017). Informe sector automotor, octubre 2017. Asociación Colombiana de Vehículos Automotores. Recuperado de http://www.andemos. org/index.php/2017/11/02/andemos-informe-sector-automotor-octubre-2017/

BBVA Research (2017). Situación automotriz, Colombia 2017. Recuperado de https://www.bbvaresearch. com/publicaciones/colombia-presentacion-situacion-automotriz-2017/

Blumer, H. (1954). What's wrong with the social theory. American Sociological Review, (19), 3-10.

Carrete y Arroyo (2013). ¿Cómo diseñar una estrategia de comunicación para vehículos eléctricos? Congreso internacional de Contaduría, Administración e Informática. Recuperado de: http:// congreso.investiga.fca.unam.mx/docs/xviii/docs/13.01.pdf

Childe, V. (1984). La evolución social. Madrid: Alianza.

Douglas, M., Isherwood, B. y Mercado, E. (1990). El mundo de los bienes. Ciudad de México: Grijalbo.

Durán, D., Chacón, S., Ortegón, L. y Atehortúa, C. (2014). Valor percibido de vehículos de lujo: dimensionalidad e implicaciones en mercadeo (Tesis de maestría). Institución Universitaria Politécnico Grancolombiano, Bogotá, Colombia. Recuperado de http://190.131.241.186/ handle/10823/749 
Duque, P. (2014). Neuromarketing una herramienta validadora en la toma de decisiones en mercadeo visual-auditivo (Tesis de maestría). Universidad Nacional de Colombia, Manizales. Recuperado de http://www.bdigital.unal.edu.co/40204/

Durkheim, E. (1986). Las reglas del método sociológico. Ciudad de México: Fondo de Cultura Económica.

El Colombiano. (2016). Crisis ambiental en el aburrá. Recuperado de http://www.elcolombiano.com/ opinion/editoriales/crisis-ambiental-en-el-aburra-BJ3856416

El Colombiano. (2017) Todavía hay tiempo para mejorar la movilidad. Recuperado de http://www. elcolombiano.com/antioquia/movilidad/todavia-hay-tiempo-para-mejorar-la-movilidad-BC7703762

Graves-Brown, P.M. (2000). Matter, Materiality and Modern Culture. Londres: Routledge.

Giucci, G. (2007). La vida cultural del automóvil. Bernal: Univ. Nacional de Quilmes.

Kaufmann, V. (2002). Re thinking mobility. Aldershot: Ashgate.

Hennessey, A (2014) Estrategias De Mercadeo Para Impulsar La Comercialización De Carros Eléctricos En Bogotá, Universidad Militar Nueva Granada.

McCracken, G. (1986). Culture and Consumption: A Theoretical Account of the Structure and Movement of the Cultural Meaning of Consumer Goods. The Journal of Consumer Research, 13, 71-84.

Martín Juez, F. (2002). Contribuciones para una antropología del diseño. Barcelona: Editorial Gedisa S.A.

Martínez Miguélez, M. (2006). Ciencia y arte en la metodología cualitativa. Ciudad de México: Trillas.

Mead, G. H. (1934). Mind, self and society: from the Standpoint of a Social Behaviorist. Chicago: University of Chicago Press.

Moscovici, S. y Duveen, G. (2000). Social representations: explorations in social psychology. Cambridge: Polity.

Morales Quintana, B (2014), Modelo de masificación de vehículos eléctricos en Bogotá D.C. Bogotá D.C., Colombia. Universidad Nacional de Colombia, Departamento de Ingeniería Eléctrica y Electrónica

Programa Medellín Cómo Vamos. (2016). Informe de Calidad de Vida de Medellín 2012-2015. Recuperado de https://www.medellincomovamos.org/download/informe-de-indicadores-objetivossobre-la-calidad-de-vida-en-medellin-2012-2015/

Sahlins, M. (1977) Economía de la edad de piedra. Madrid: Akal Editor.

Sheller, M. (2004). Automotive emotions: feeling the car. Theory, Culture y Society, 21, 25-39.

Urry, J. (2003). Inhabiting the Car. Department of Sociology at Lancaster University. Recuperado de http:// www.lancaster.ac.uk/fass/resources/sociology-online-papers/papers/urry-inhabiting-the-car.pdf

Urry, J. (2004) "The 'System' of Automobility", Revista Theory, Culture y Society, Volumen 21, p 221 242, Lóndres, Inglaterra.

Vallejo, F. y López, Z. (2016). Entrevista al ingeniero Jorge Barrera. Recuperada de https://youtu. be/lfesqdY2u3I 